\title{
A stochastic evaluation method based on the least en- tropy criterion for room with arbitrary environmental noise excitation
}

\author{
Mitsuo Ohta* and Yoshifumi Fujita** \\ *Department of Electronic and Information Engineering, \\ Faculty of Engineering, Kinki University, \\ Umenobe 1, Takaya, Higashi-Hiroshima, 729-17 Japan \\ **Department of Mechanical Engineering, Kure National College of Technology, \\ Aga-Minami 2-2-11, Kure, 737 Japan
}

(Received 2 December 1992)

\begin{abstract}
As one of evaluation methods for the room sound fluctuation, a new trial to predict the probability distribution of the response sound fluctuation in the room excited by arbitrary type environmental noise input is proposed by emphasizing its methodological viewpoint. The response sound intensity of the room can be naturally expressed as a total sum on intensity scale of each frequency response component and its modelling error caused by the quantized error and/or the observation noise. Owing to the nonGaussian property of intensity variable and modelling error, the well-known system identification method is too difficult to be directly applied to the present case. So, in this paper, first by newly introducing some kind of information measure in a form of mutual entropy quantity between input and output and maximizing it, a new estimation algorithm of acoustic characteristic parameters of the room is derived by applying a stochastic approximation method to the above extremal condition with using a general expression form of the Gram-Charier series expansion type non-Gaussian probability distribution. In the room excited by an arbitrary environmental noise input fluctuating in non-Gaussian type probability distribution form, the probability distribution of response sound fluctuation for an overall frequency range and each frequency band component can be predicted by using the above identified system model. The proposed method is applied to the room simulating the actual living acoustic situation and its justification and usefulness is experimentally confirmed, too.
\end{abstract}

Keywords: Stochastic acoustic evaluation, Non-Gaussian acoustic signal, Least entropy criterion, Stochastic approximation method, System identification

PACS number: 43. 55. Cs, 43. 55. Nd, 43. 55. $\mathrm{Br}$

\section{INTRODUCTION}

It is important for the stochastic evaluation of acoustic living environment to predict minutely the probabilistic response fluctuation of a room excited by an arbitrary environmental sound input of nonGaussian distribution type. Needless to say, owing to this non-Gaussian property, the well-known standard evaluation method based only on statistical mean and variance is not sufficient to evaluate its whole fluctuation characteristic in the room, and it seems to be necessary as one of stochastic evaluation methods to newly find the corresponding response probability distribution. In this paper, a new method of predicting this whole probability distribution form of response fluctuation is proposed especially by emphasizing its methodological viewpoint. 
First, the response sound intensity of the room excited by arbitrary type environmental noise input can be naturally expressed by a total sum on intensity scale of each frequency response component and its modelling error and/or observation noise. Then, the estimation problem for the system parameters is concretely considered under introducing some error criterion. However, the application of well-known least squares error criterion is not sufficient for evaluating the whole response probability distribution form showing the non-Gaussian characteristic, and so some other kinds of estimation criterion should be introduced. For this purpose, as one of error evaluation criteria, some kind of information measure based on the mutual entropy quantity between input and output is newly introduced and then maximized. More concretely, after employing the stochastic approximation method, the recursive algorithm for the practical estimation of system parameters is derived. Next, by using this identified linear system model on energy scale for the acoustic characteristics of the room, the probability distribution of the response sound fluctuation of the room excited by an environmental random noise of arbitrary distribution type can be predicted.

Finally, by applying the proposed method to the room simulating the actual living acoustic environment, its effectiveness is confirmed experimentally too.

\section{THEORETICAL CONSIDERATION}

As mentioned in the introduction, the acoustic input-output relation of the room excited by an environmental noise can be directly expressed in the following expression form:

$$
y_{k}=\sum_{i=1}^{m} a_{i} x_{i k}+v_{k},
$$

where $x_{i k}$ [Watt] is a value of the divided component existing in the $i$-th frequency band for an input sound power fluctuation at the $k$-th sampling time when the overall frequency range of input expands over $m$ component frequency bands. Also, $y_{k}$ [Watt $\left./ \mathrm{m}^{2}\right]$ is the output sound intensity fluctuation at the $k$-th sampling time, and $a_{i}\left[1 / \mathrm{m}^{2}\right]$ is the $i$-th characteristic parameter related to an averaged absorption coefficient of the room in the $i$-th frequency band. $v_{k}\left[\mathrm{Watt} / \mathrm{m}^{2}\right]$ is also an observation noise and/or modelling error and fluctuates station- arily in non-Gaussian type distribution form. From the object of present study to predict the intensity response probability distribution of the room, first, it becomes essential to estimate only the above characteristic parameter $a_{i}$. Instead of using the wellknown least squares error criterion method, ${ }^{1)}$ the mutual entropy information criterion between input and output ${ }^{2)}$ which can treat universally the nonGaussian property of $v_{k}$ is introduced as follows:

$$
I\left(\boldsymbol{x}_{k}, y_{k}\right)=H\left(y_{k}\right)-H\left(y_{k} \mid \boldsymbol{x}_{k} ; \boldsymbol{a}\right),
$$

where $H\left(y_{k}\right)$ is an entropy of output $y_{k}$ and $H\left(y_{k} \mid \boldsymbol{x}_{k} ; \boldsymbol{a}\right)$ is the conditional entropy. That is, by maximizing this $I\left(\boldsymbol{x}_{k}, y_{k}\right)$, the optimum value of $a_{i}$ can be estimated. Here, maximizing $I\left(\boldsymbol{x}_{k}, y_{k}\right)$ is equivalent to minimizing the conditional entropy $H\left(y_{k} \mid \boldsymbol{x}_{k} ; \boldsymbol{a}\right)$ with respect to parameter $\boldsymbol{a}$ defined as

$$
\begin{aligned}
H\left(y_{k} \mid \boldsymbol{x}_{k} ; \boldsymbol{a}\right) & =-\iint p\left(\boldsymbol{x}_{k}, y_{k}\right) \log p\left(y_{k} \mid \boldsymbol{x}_{k}\right) d \boldsymbol{x}_{k} d y_{k} \\
& =-\iint p\left(\boldsymbol{x}_{k}, y_{k}\right) \log p_{v}\left(v_{k}\right) d \boldsymbol{x}_{k} d y_{k}
\end{aligned}
$$

where $p_{v}(v)$ is the probability density function of $v_{k}$. Minimizing Eq. (3) is reduced to the following extremal condition:

$$
\begin{aligned}
\frac{\partial H\left(y_{k} \mid \boldsymbol{x}_{k} ; \boldsymbol{a}\right)}{\partial a_{i}} & =-\iint \frac{p\left(\boldsymbol{x}_{k}, y_{k}\right)}{p_{v}\left(v_{k}\right)} \frac{\partial p_{v}\left(v_{k}\right)}{\partial a_{i}} d \boldsymbol{x}_{k} d y_{k} \\
& =-\left\langle\frac{1}{p_{v}\left(v_{k}\right)} \frac{\partial p_{v}\left(y_{k}\right)}{\partial a_{i}}\right\rangle=0 .
\end{aligned}
$$

Before deriving the recurrence type parameter estimation algorithm, the expression form of the probability density function of $v_{k}$ of non-Gaussian type should be noticed to be given in advance. That is, as a general expression of $P_{v}(v)$, the following Gram-Charier A type series expansion ${ }^{3)}$ can be chosen:

$$
\begin{aligned}
p_{v}(v)= & \frac{1}{\sqrt{2 \pi} \sigma_{v}} \exp \left(-\frac{\left(v-\mu_{v}\right)^{2}}{2 \sigma_{v}{ }^{2}}\right) \\
& \cdot \sum_{n=0}^{\infty} \frac{\alpha_{n}}{\sqrt{n !}} H_{n}\left(\frac{v-\mu_{v}}{\sigma_{v}}\right) .
\end{aligned}
$$

Then, after substituting this equation into Eq. (4), by applying the stochastic approximation method, ${ }^{4,5)}$ the recurrence estimation algorithm is derived as follows: 


$$
\begin{aligned}
\hat{\boldsymbol{a}}^{(k)}=\hat{\boldsymbol{a}}^{(k-1)}-\boldsymbol{\Gamma}_{k} \frac{\boldsymbol{x}_{k}}{\sigma_{v}} & \\
& \frac{\sum_{n=0}^{\infty} \frac{\alpha_{n}}{\sqrt{n !}} H_{n+1}\left(\frac{y_{k}-\sum_{i=1}^{m} \hat{a}_{i}^{(k-1)} x_{i k}-\mu_{v}}{\sigma_{v}}\right)}{\sum_{n=0}^{\infty} \frac{\alpha_{n}}{\sqrt{n !}} H_{n}\left(\frac{y_{k}-\sum_{i=1}^{m} \hat{a}_{i}^{(k-1)} x_{i k}-\mu_{v}}{\sigma_{v}}\right)},
\end{aligned}
$$

where,

$$
\begin{aligned}
\mu_{v} & =\left\langle y_{k}-\sum_{i=1}^{m} \hat{a}_{i}^{(k-1)} x_{i k}\right\rangle, \\
\sigma_{v}{ }^{2} & =\left\langle\left(y_{k}-\sum_{i=1}^{m} \hat{a}_{i}^{(k-1)} x_{i k}-\mu_{v}\right)^{2}\right\rangle, \\
\alpha_{n} & =\frac{1}{\sqrt{n !}}\left\langle H_{n}\left(\frac{y_{k}-\sum_{i=1}^{m} \hat{a}_{i}^{(k-1)} x_{i k}-\mu_{v}}{\sigma_{v}}\right)\right\rangle
\end{aligned}
$$

and

$$
\Gamma_{k}=\operatorname{diag}\left(\gamma_{1}^{(k)}, \cdots, \gamma_{m}^{(k)}\right) .
$$

Here, the notation $\langle>$ means an expectation, $H_{n}(\quad)$ is an Hermite polynomial of the $n$-th order and $\Gamma_{k}$ is a diagonal matrix satisfying the following Robbins-Monro's condition:

$$
\begin{gathered}
\gamma_{i}^{(k)}>0, \\
\sum_{k=1}^{\infty} \gamma_{i}^{(k)}=\infty
\end{gathered}
$$

and

$$
\sum_{k=1}^{\infty}\left(\gamma_{i}^{(k)}\right)^{2}<\infty .
$$

Needless to say, for ensuring the convergence condition of the above algorithm in Eq. (6), RobbinsMonro's condition should be satisfied. Now, by using the estimated values $\hat{a}_{i}$ 's of characteristic parameters based on the algorithm in Eq. (6), the probability distribution of the response sound energy density can be predicted in the actual case when the room is excited by the different type arbitrary environmental random noise fluctuating in an arbitrary non-Gaussian type probability distribution form. The probability distribution corresponding to the random input of an overall frequency range and each frequency component within only the $i$-th frequency band can be easily predicted by employing the following Eq. (9) and Eq. (10) respectively:

$$
y_{k}=\sum_{i=1}^{m} \hat{a}_{i} x_{i k},
$$

$$
y_{k}=\hat{a}_{i} x_{i k} .
$$

\section{EXPERIMENTAL CONSIDERATION}

The experiments have been performed in a reverberation room (volume $189.2 \mathrm{~m}^{3}$, surface 197.2 $\left.\mathrm{m}^{2}\right)$ with 3 bottom boards of home bed $(0.9 \times 0.93$ $\mathrm{m}^{2}$ ) as the absorption material in order to simulate the actual living situation as really as possible. Figure 1 shows its experimental setup. Nondirectional speaker and microphone have been arranged such that the distance between them was $100 \mathrm{~cm}$, the height of speaker from the floor was $60 \mathrm{~cm}$ and the height of microphone from the floor was $85 \mathrm{~cm}$. Here, we must notice the fact that especially in a room producing the non-diffuse sound field, the values of its characteristic parameter $a_{i}$ 's depend on the location of speaker and microphone.

The main purpose of this study is focussed on a new proposal of methodology and experimental confirmation of its principle on the probability distribution for the stochastic fluctuation of room sound. Furthermore, as is well known, the response sound fluctuation of a room especially for the high frequency part of each of road traffic noise and music sound noise can not originally have effectively wide amplitude fluctuation width of randomness. From above reasons, only the low frequency part which expresses a large ups and downs in the response sound fluctuation has been intentionally chosen as the object of this study. For this case, 1/1 octave

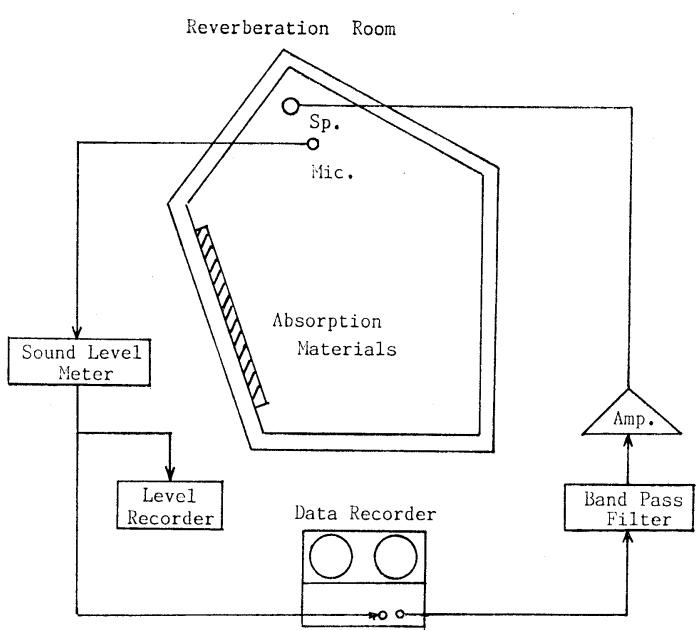

Fig. 1 Experimental setup. 
frequency band from 90 to $180 \mathrm{~Hz}$ has been used as an input overall frequency range and it has been divided into 3 frequency components by using a $1 / 3$ octave band pass filter.

First, by employing a white noise, through a $1 / 1$ octave band pass filter from 90 to $180 \mathrm{~Hz}$, generated by noise generator, the characteristic parameters of this room have been identified by using the proposed estimation method. Here, in order to measure $x_{i k}$ 's $(i=1,2,3)$ especially, which are the sound power components corresponding to each of $1 / 3$ octave frequency bands from 90 to $112 \mathrm{~Hz}$, from 112 to $140 \mathrm{~Hz}$ and 140 to $180 \mathrm{~Hz}$, in an anechoic room, the signal of the input random noise recorded by a data recorder, through a $1 / 3$ octave band pass filter under the same relative arrangement between speaker and microphone as in objective room, has been regenerated and measured by sound level meter in advance. For each frequency band component of the random input and an overall frequency range of the output, we have sampled 500 data at every $0.5 \mathrm{~s}$ interval simultaneously. At this case, modelling error $v_{k}$ has been calculated by substituting values of $x_{1 k}, x_{2 k}, x_{3 k}$ and $y_{k}$ and recursively estimated values of $\hat{a}_{i}^{(k-1)}$ 's into $a_{i}$ of Eq. (1) and then its statistical values have been recursively evaluated in advance. The estimated result of system parameters is shown in Figs. 2, 3 and 4. Here, $a_{1}, a_{2}$, $a_{3}$ are three system parameters corresponding to each $1 / 3$ octave frequency bands from 90 to $112 \mathrm{~Hz}$, from 112 to $140 \mathrm{~Hz}$ and from 140 to $180 \mathrm{~Hz}$ respectively. Initial conditions, a gain matrix in calculation of the above recursive algorithm Eq. (6) and the estimated values are shown in Table 1. According to the initial conditions, the estimated values converge to slightly different ones. Next, by regarding Eq. (1) with no additive modelling error as theoretical model of the room characteristics, the probability distribution form of the sound intensity response fluctuation in the room has been concretely predicted when the room is excited by each of the actual traffic noise and music sound (i.e., Karaoke noise) through a $1 / 1$ octave band pass filter, of which the probability distribution on energy scale is not expressed in Gaussian distribution form but in nonGaussian distribution form. Before computing by use of the above identified model, the $1 / 3$ octave frequency band components from 90 to $112 \mathrm{~Hz}$, from 112 to $140 \mathrm{~Hz}$ and from 140 to $180 \mathrm{~Hz}$ of each input have been measured in the same situation as stated in the experiment of identification for the room characteristic parameters. Figures 5 and 6

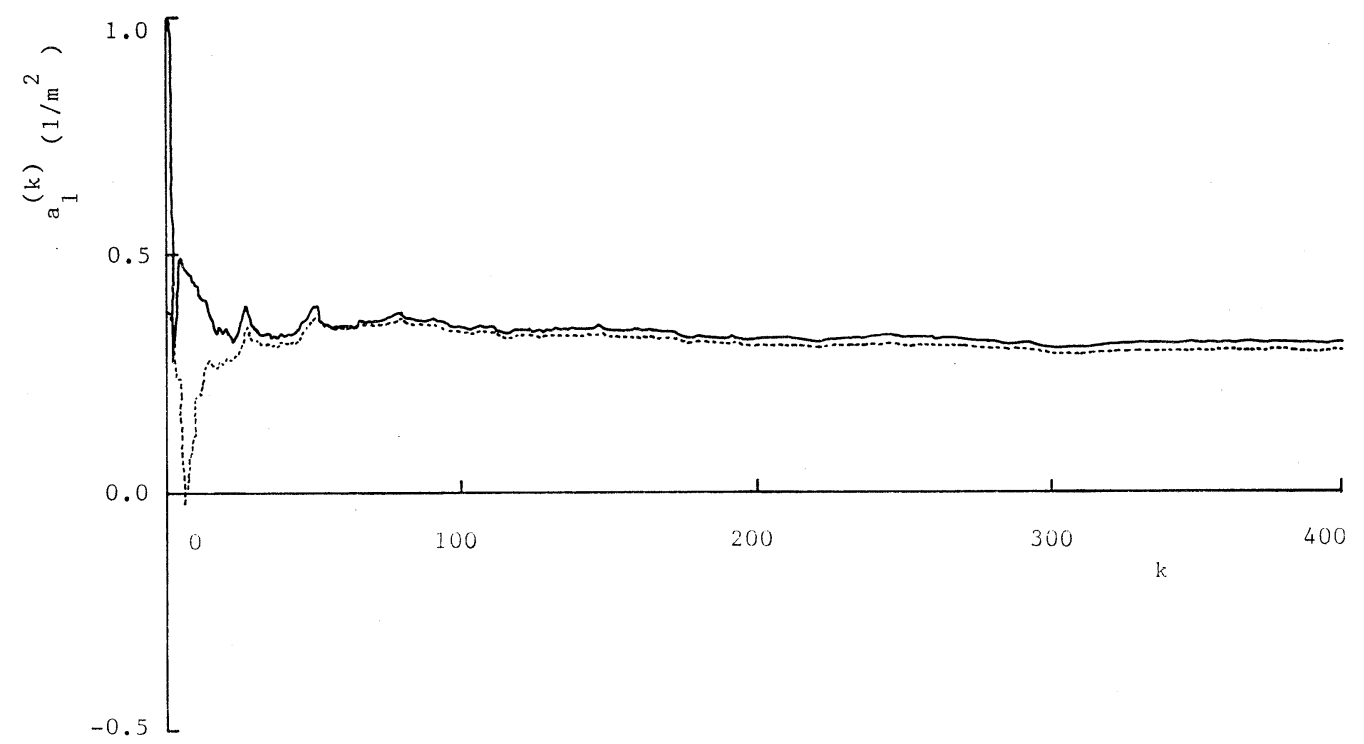

Fig. 2 Temporal behavior of recursive estimated value $\hat{a}_{1}{ }^{(k)}$ of a characteristic parameter $a_{1}$ of the room corresponding to two different initial conditions shown in Table 1. A dotted line shows the result corresponding to case (i) and a solid line shows the result corresponding to case (ii). 


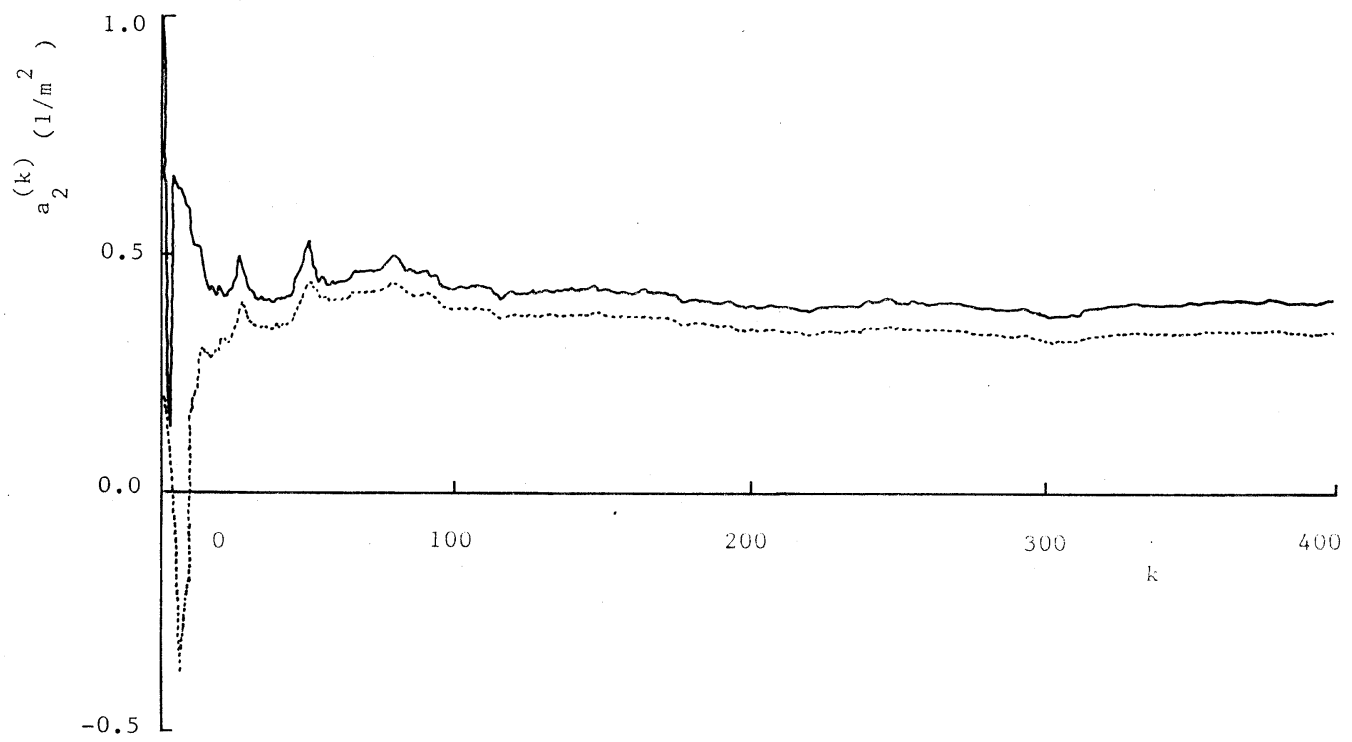

Fig. 3 Temporal behavior of recursive estimated value $\hat{a}_{2}{ }^{(k)}$ of a characteristic parameter $a_{2}$ of the room corresponding to two different initial conditions shown in Table 1 . A dotted line shows the result corresponding to case (i) and a solid line shows the result corresponding to case (ii).

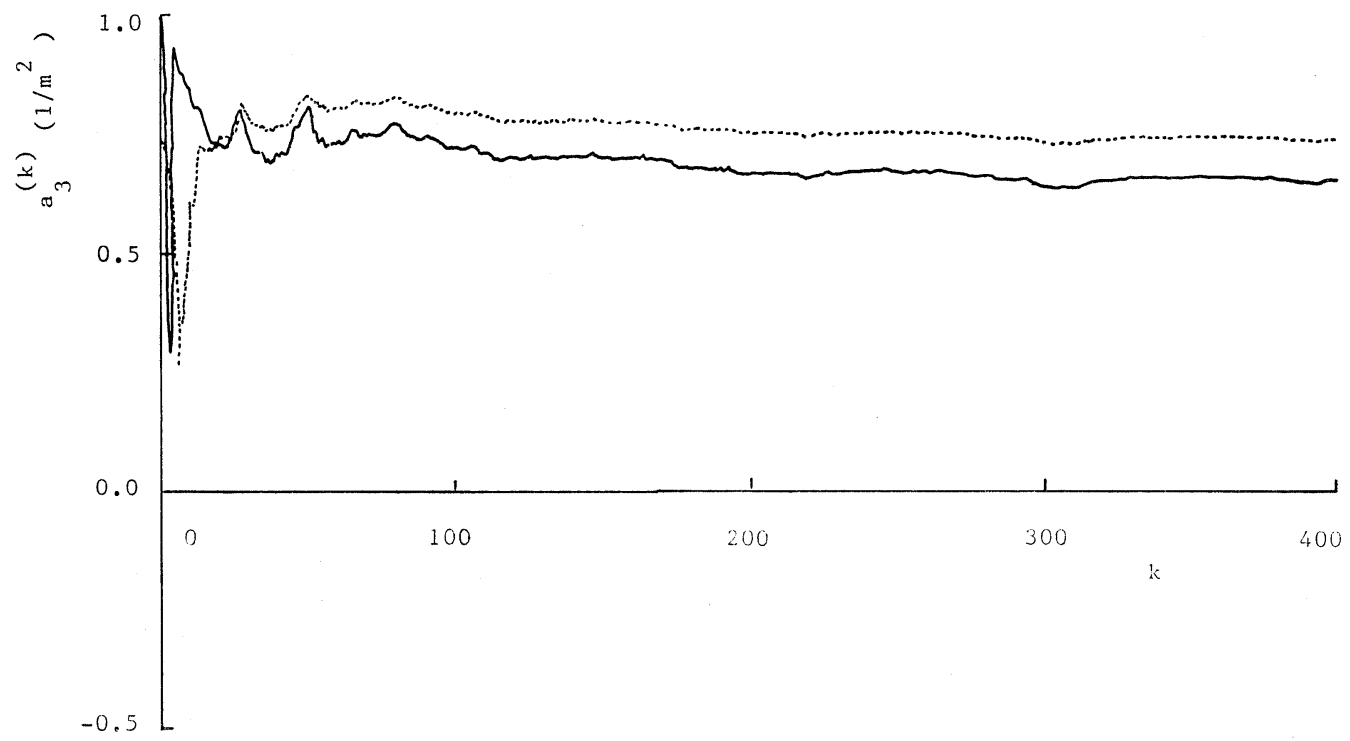

Fig. 4 Temporal behavior of recursive estimated value $\hat{a}_{3}{ }^{(k)}$ of a characteristic parameter $a_{3}$ of the room corresponding to two different initial conditions shown in Table 1. A dotted line shows the result corresponding to case (i) and a solid line shows the result corresponding to case (ii).

show a comparison between theoretical cumulative probability distribution and experimentally sampled values for two cases with an overall traffic noise input and with only a $1 / 3$ octave band component from 140 to $180 \mathrm{~Hz}$. Figures 7 and 8 show a comparison between theoretical cumulative probability 
Table 1 Initial conditions and gain matrix in the proposed estimation method and corresponding estimated values.

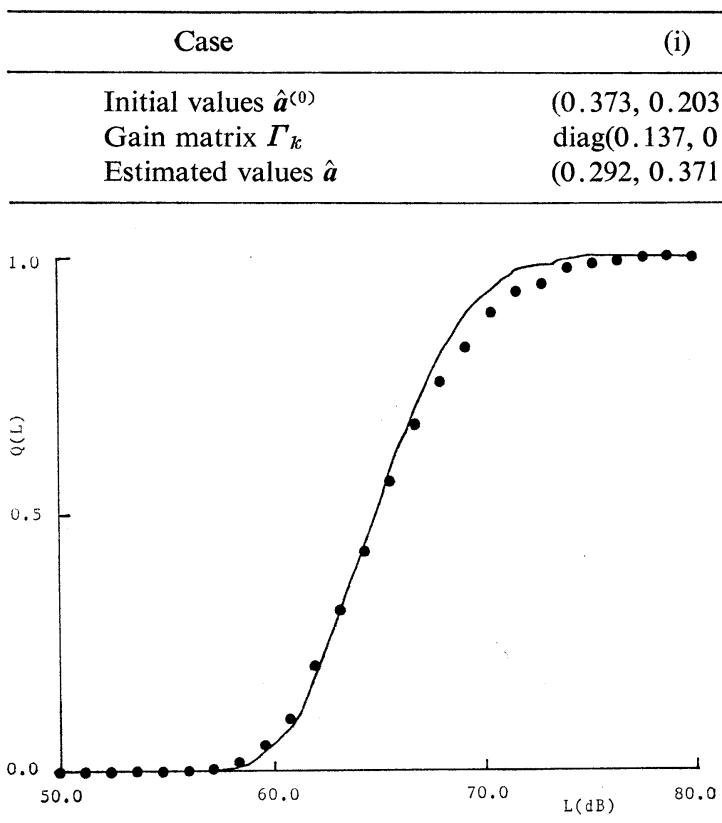

Fig. 5 A comparison between theoretically predicted curve (solid line) and experimentally sampled values (dotted points), for the cumulative probability distribution form of room response with an overall traffic noise input.

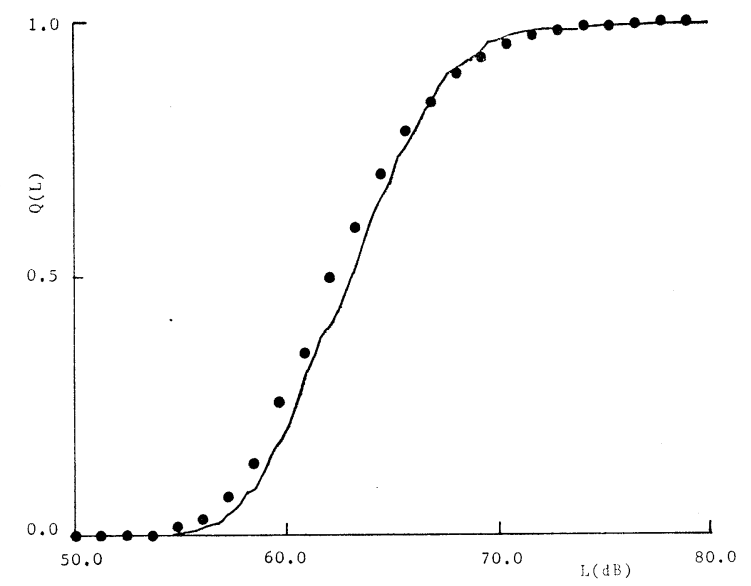

Fig. 6 A comparison between theoretically predicted curve (solid line) and experimentally sampled values (dotted points), for the cumulative probability distribution form of room response with a $1 / 3$ octave band component input, from 140 to $180 \mathrm{~Hz}$, in the case with the traffic noise excitation.
$(1,1,1)$

$\operatorname{diag}(0.173,0.175,0.179) / k$

$(0.334,0.410,0.635)$

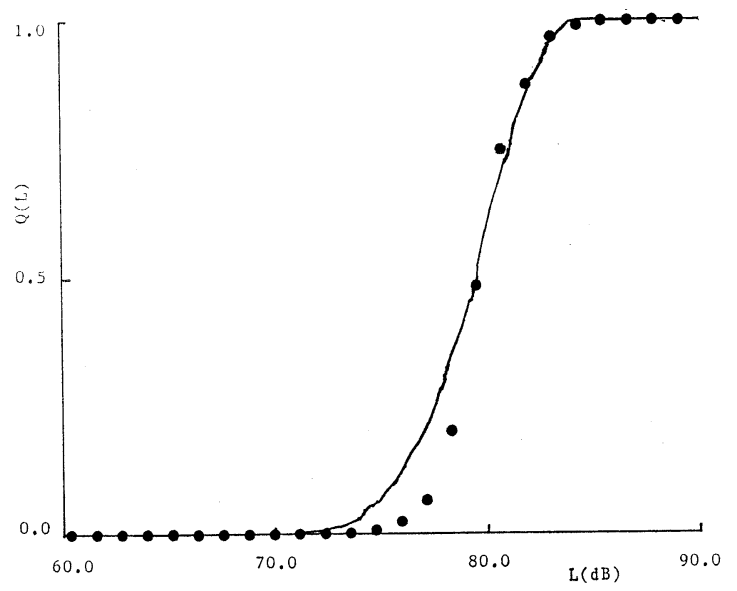

Fig. 7 A comparison between theoretically predicted curve (solid line) and experimentally sampled values (dotted points), for the cumulative probability distribution form of room response with an overall music sound input.

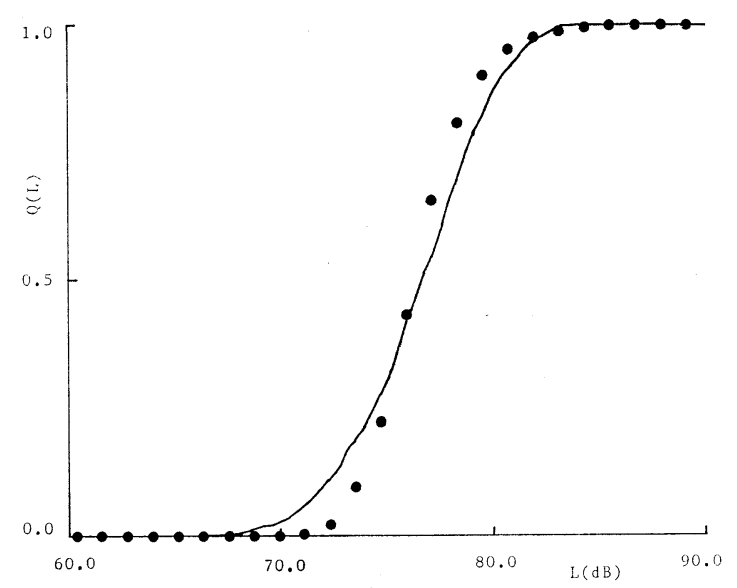

Fig. 8 A comparison between theoretically predicted curve (solid line) and experimentally sampled values (dotted points), for the cumulative probability distribution form of room response with a $1 / 3$ octave band component input, from 140 to $180 \mathrm{~Hz}$, in the case with the music sound excitation. 
distribution and experimentally sampled values for two cases with an overall music sound input and with only a $1 / 3$ octave band component from 140 to $180 \mathrm{~Hz}$. Here, it is so difficult to derive the theoretical expression of the response probability distribution based on Eq. (9) and Eq. (10) owing to nonGaussian property of traffic noise on energy scale that theoretical cumulative distribution curves in Fig. 5 and Fig. 6 have been plotted by employing Eq. (9) and Eq. (10) with the estimated values of $a_{i}$ 's for case (ii) in Table 1, respectively, calculating $y_{k}$ corresponding to 500 data of three divided $1 / 3$ octave frequency band components of the input sampled at every $0.5 \mathrm{~s}$ interval and getting the cumulative relative frequency distribution of $y_{k}$. Theoretical cumulative distribution curves in Fig. 7 and Fig. 8 have been plotted by above similar procedure with use of Eq. (9) and Eq. (10), respectively.

\section{CONCLUSION}

In this paper, a new stochastic evaluation method has been proposed predicting the probability distribution form of the response sound intensity fluctuation in the room excited by the arbitrary type environmental random noise fluctuating in a nonGaussian distribution form. In the proposed method, it is possible to predict the response probability distribution of a room for both cases with an overall frequency range and with an each frequency band component for the actual stochastic input. The usefulness of the proposed method has been experimentally confirmed, too.

\section{ACKNOWLEDGEMENT}

We would like to express our cordial thanks to Mr. H. Masuda for his helpful assistance and much discussion in 1991 International Conference on Noise Control Engineering. ${ }^{6)}$

\section{REFERENCES}

1) P. Eykhoff, System Identification (John Wiley \& Sons, London, 1974), p. 39.

2) H. L. Weidmann and E. B. Stear, "Entropy analysis of parameter estimation," Inf. Control 14, 493506 (1969).

3) M. Ohta and T. Koizumi, "General statistical treatment of the response of a nonlinear rectifying device to a stationary random input," IEEE Trans. Inf. Theory IT-14, 595-598 (1968).

4) H. Robbins and S. Monro, "A stochastic approximation method," Ann. Math. Stat. 22(1), 400-
407 (1951).

5) A. P. Sage and J. L. Melsa, System Identification (Academic Press, New York, 1971), p. 112.

6) M. Ohta and Y. Fujita, "A response probability evaluation method for the acoustic system with arbitrary type noise excitation based on least entropy criterion," Proc. Inter-Noise 91 (1), 475478 (1991).

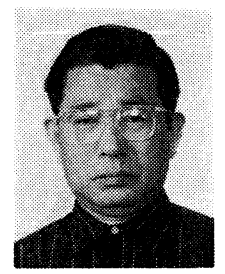

Mitsuo Ohta (age 65) $\quad$ B.Sc. (1952) and D.Sc. (1961) degrees from Kyoto Univ. (Dept. of Physics), associate professors (1959: Dept. of Measurement, Kobe Univ. and 1963: Dept. of Applied Physics, Fukui Univ.), professors (1967: Dept. of Applied Physics and 1968: Dept. of Electronics, Fukui Univ.; 1969: Depts. of Electricity and 1975: Circuit and System Engineerings, Hiroshima Univ.; 1990 Dept. of Computer \& Electronics, Kinki Univ.), honorary prof. (1990: Hiroshima Univ.), prize in praise of paper (1966: Acoustical Society of Japan), licence of authorized technical expert (1987: Institute of Noise Control Engineers of Japan), members of Acoustical Society, Institute of Electronics, Information and Communication, Institute of Electrical Engineers, Institute of Systems, Control and Information Engineers, Institute of Noise Control Engineers, Society of Instrument and Control Engineers of Japan. His research fields have been microwave, dielectric measurement, ultra-sound, electro-magneticwave propagation, information processing, control and probabilistic engineering, sound and vibration control, digital filter and others.

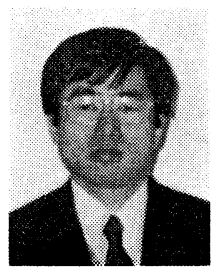

Yoshifumi Fujita was born in Yamato-Kohriyama, Nara, on January 16, 1950. He received the B.E. degree from Kyoto Institute of Technology in 1974 and the M.E. degree from Kyoto University in 1976. From 1976 to 1982 , he was an Assistant, and from 1982 to 1987 he was a Lecturer, and from 1987 to 1993 he was an Assistant Professor at the Department of Mechanical Engineering, Kure National College of Technology. Since 1993 he has been an Associate Professor at the Department of Management and Information Science, Onomichi Junior College. His current research interests lie in signal processing of random noise, digital control by microcomputer and computer-aided control system design. He is a member of the Acoustical Society of Japan, the Institute of Systems, Controls and Information Engineers of Japan, the Institute of Noise Control Engineers, the Society of Instrument and Control Engineers of Japan, the Institute of Electronics, Information and Communication, the Institute of Electrical Engineers of Japan. 